\title{
Soluções irrigadoras utilizadas no preparo químico-mecânico do sistema de canais radiculares: uma revisão da literatura
}

\author{
Irrigation solutions used in the chemical-mechanical preparation of the root canals system: a
} literature review

Soluciones de riego utilizadas en la preparación químico-mecánica del sistema de conductos radiculares: una revisión de la literatura

\section{Resumo}

O presente trabalho tem como objetivo revisar a literatura identificando a importância dos compostos químicos utilizados no preparo biomecânico do sistema de canais radiculares, comparando os compostos mais utilizados na prática clínica para a realização da etapa operatória de irrigação. Foi realizado um levantamento bibliográfico nas bases de dados do PubMed, Scielo, Lilacs, Science Direct e consultas em livros na biblioteca física de uma faculdade privada, sendo incluídos estudos que abordassem a irrigação no tratamento dos sistema de canais radiculares, com delimitação de período de tempo específico entre 2013 a 2021, e excluídos aqueles sem relação direta com o assunto. Seguindo os critérios de inclusão, foi realizado a leitura dos resumos disponíveis e foram identificados 20 artigos, os quais fizeram parte do presente estudo. Diante do exposto, cada substância irrigante estudada, apresenta suas vantagens e desvantagens, sendo o Hipoclorito de Sódio $(\mathrm{NaOCl})$ o irrigante mais empregado por demostrar ação antimicrobiana e a capacidade de dissolver tecido orgânico, proporcionando o sucesso no tratamento do SCR, entretanto, em concentrações elevadas pode apresentar citotoxidade aos tecidos pulpares e periradiculares. Dessa forma, torna-se de fundamental importância o conhecimento dos profissionais a respeito dessas soluções na sua utilização, com o propósito de compreender os resultados que serão alcançados na etapa operatória de irrigação.

Palavras-chave: Clorexidina; Hipoclorito de sódio; Preparo de canal radicular; EDTA; Endodontia.

\begin{abstract}
The present work aims to review the literature identifying the importance of chemical compounds used in the biomechanical preparation of the root canal system, comparing the compounds most used in clinical practice to carry out the operative stage of irrigation. A bibliographic survey was carried out in the databases of PubMed, Scielo, Lilacs, Science Direct and book queries in the physical library of a private college, including studies that addressed irrigation in the treatment of root canal systems, with a delimited period of specific time between 2013 to 2021 , excluding those with no direct relation to the subject. Following the inclusion criteria, the available abstracts were read and 20 articles were identified, which were part of this study. Given the above, each irrigating substance studied has its advantages and disadvantages, with Sodium Hypochlorite $(\mathrm{NaOCl})$ being the most used irrigant for demonstrating antimicrobial action and the ability to dissolve organic tissue, providing success in the treatment of SCR, however, in high concentrations may present cytotoxicity to pulp and periradicular tissues. Thus, the knowledge of professionals about these solutions in their use is of fundamental importance, in order to understand the results that will be achieved in the operative stage of irrigation.
\end{abstract}

Keywords: Chlorhexidine; Sodium hypochlorite; Root canal preparation; EDTA; Endodontics. 


\begin{abstract}
Resumen
El presente trabajo tiene como objetivo revisar la literatura identificando la importancia de los compuestos químicos utilizados en la preparación biomecánica del sistema de conductos radiculares, comparando los compuestos más utilizados en la práctica clínica para llevar a cabo la etapa operativa del riego. Se realizó un levantamiento bibliográfico en las bases de datos de PubMed, Scielo, Lilacs, Science Direct y consultas de libros en la biblioteca física de un colegio privado, incluyendo estudios que abordaron el riego en el tratamiento de los sistemas de conductos radiculares, con un período delimitado de tiempo específico. entre 2013 y 2021, excluyendo aquellos sin relación directa con el tema. Siguiendo los criterios de inclusión, se leyeron los resúmenes disponibles y se identificaron 20 artículos, que formaron parte de este estudio. Dado lo anterior, cada sustancia de riego estudiada tiene sus ventajas y desventajas, siendo el hipoclorito de sodio $(\mathrm{NaOCl})$ el irrigante más utilizado para demostrar la acción antimicrobiana y la capacidad de disolver tejidos orgánicos, proporcionando éxito en el tratamiento de la SCR, sin embargo, en altas concentraciones. puede presentar citotoxicidad a los tejidos pulpares y perirradiculares. Por ello, el conocimiento de los profesionales sobre estas soluciones en su uso es de fundamental importancia, a fin de comprender los resultados que se alcanzarán en la etapa operativa del riego.
\end{abstract}

Palabras clave: Clorhexidina; Hipoclorito de sódio; Preparación del conducto radicular; EDTA; Endodoncia.

\title{
1. Introdução
}

O Tratamento Endodôntico (TE) é um procedimento realizado com o objetivo de proporcionar o reparo tecidual pósterapia de modo a conduzir à remissão da lesão periapical. Este tratamento constitui-se na desinfecção do sistema de canais radiculares (SCR) e na prevenção de uma nova reinfecção, por meio da eliminação dos microrganismos, do tecido necrótico e pulpar, identificado no interior do canal radicular. Em vista disso, a remoção e desinfecção do SCR são etapas essenciais, e tem como intuito garantir o sucesso na realização do tratamento (Siqueira et al., 2014; Souza, 2015; Rahimi et al., 2014).

Os microrganismos constituem o principal agente etiológico das patologias pulpares e perirradiculares, com predominância das espécies anaeróbicas obrigatórias principalmente as gram-negativas, que desempenham um importante papel na indução e duração de processos inflamatórios. Entretanto, as bactérias facultativas como o enterococcus faecalis são as responsáveis pelas falhas que ocorre no TE. Dessa maneira, a eliminação dos microrganismos depende de vários requisitos, tais como uma instrumentação adequada, uma irrigação efetiva e por fim uma obturação tridimensional que promova um selamento completo do canal radicular (Dioguardi et al., 2018).

A instrumentação e a irrigação do SCR devem ser realizadas simultaneamente, formando o preparo químico-mecânico (PQM). Este preparo exerce um importante passo na limpeza e desinfecção dos canais, como também na sua modelação, preparando-os para receberem o selamento tridimensional. O preparo mecânico por si só não é suficiente para eliminar os microrganismos presente nos canais radiculares, sendo necessário recorrer à ajuda de uma substância química com ação antimicrobiana (Gonçalves, Rodrigues, Andrade, Soares, \& Vettore, 2016; Rahimi et al., 2014).

A irrigação do SCR com um agente químico retrata uma das etapas mais importantes e críticas na terapia endodôntica, sobretudo no que diz respeito à erradicação dos microrganismos. O SCR apresenta áreas de difícil acesso pelos instrumentos, sendo as substâncias irrigadoras o único meio capaz de alcançar essas áreas. Estas substâncias são capazes de provocar a morte dos microrganismos, ajudando na sua remoção do interior dos canais, além disso, removem o tecido necrosado, inflamado e os restos dentinários, devendo ser utilizadas durante e após a instrumentação (Luz et al., 2019; Seelan et al., 2015).

As soluções irrigadoras, devem ter como objetivo proporcionar um potencial de dissolução adequada e associada a uma baixa tensão superficial para proporcionar a limpeza, não só do canal principal, mas também dos secundários, laterais, acessórios e até mesmo, dos túbulos dentinários. Em vista disso, muitas soluções irrigadoras foram propostas ao longo dos anos, dentre elas: o MTAD, QMix, SmearClear e o peróxido de hidrogénio (Dhawan et al., 2019; Ozkan, Cobankara, \& Ozer, 2020). Embora, as substâncias químicas mais utilizadas na etapa operatória de irrigação para a desinfecção dos canais radiculares são Hipoclorito de Sódio ( $\mathrm{NaOCl})$, Clorexidina ( $\mathrm{CHX}$ ) e o Ácido Etilenodiaminico Tetra-Acético (EDTA), por apresentarem propriedades interessantes e positivas ao tratamento. Entretanto, estas substâncias exibem também pontos 
negativos e controversos durante a irrigação dos canais, devido a isto, o conhecimento dos profissionais sobre os agentes irrigantes é imprescindível na terapia endodôntica (Silva, Francisco, Brum, Barbosa, \& Soares, 2016).

Dessa forma, o objetivo do presente estudo foi esclarecer, por meio de uma revisão de literatura a importância da realização da terapia dos canais radiculares, empregando as soluções irrigadoras mais utilizadas na endodontia para eliminação dos microrganismos e desinfecção do SCR, bem como analisar a ação das mesmas frente aos tecidos vivos periapicais.

\section{Metodologia}

Trata-se de uma revisão de literatura, com caráter narrativo acerca das principais soluções irrigadoras utilizadas na endodontia.

De acordo com Rother (2007) os artigos de revisão narrativa são publicações de caráter amplo, adequadas para descrever e discutir o desenvolvimento de um determinado assunto, conforme o ponto de vista teórico ou conceitual. São textos que constituem a análise da literatura científica na interpretação e análise crítica do autor.

Para elaboração do presente estudo, foi realizado um levantamento bibliográfico nas bases de dados do PubMed, Scielo, Lilacs, Science Direct e consultas em livros na biblioteca de uma Faculdade privada situada no Sudoeste da Bahia.

Para a realização de pesquisas bibliográficas em publicações online, foram utilizados descritores específicos: ("preparo de canal radicular" "clorexidina"; "hipoclorito de sódio"; "EDTA"; "endodontia" / "root canal preparation" "chlorhexidine in endodontics"; "sodium hypochlorite"; "EDTA"; "endodontics" / "; "Preparación del conducto radicular"; "clorhexidina"; "hipoclorito de sodio"; "EDTA"), juntamente com operador booleano "AND" de forma a combinar os descritores citados. De forma complementar, foi realizado pesquisas bibliográficas em publicações impressas, utilizando capítulos de livros que abordavam o tema proposto.

Foram incluídos artigos científicos e livros, com delimitação de período de tempo específico, entre 2013 a 2021, dentro da temática proposta, que abordavam a irrigação do canal radicular no tratamento endodôntico, com textos completos, disponíveis, publicados na língua portuguesa, inglesa e espanhola. Foram excluídos trabalhos de conclusão de curso, monografias, dissertações e aquelas que não atendessem aos objetivos propostos ou que não estavam de acordo aos critérios de inclusão.

Os artigos foram selecionados a partir de uma leitura criteriosa, e posteriormente com a elaboração de fichamentos. A partir disso, foi realizada uma análise narrativa dos artigos, na qual foram ordenados de modo a construir conhecimento a respeito das soluções irrigadoras mais utilizadas no preparo químico mecânico.

\section{Revisão da Literatura}

\subsection{Fatores Importantes para o Sucesso da Terapia Endodôntica}

$\mathrm{Na}$ endodontia, o conhecimento acerca da anatomia interna dos canais radiculares, bem como a limpeza e modelagem são fatores determinantes para obter êxito no TE. O profissional deve realizar o diagnóstico corretamente para identificar possíveis particularidades no elemento dentário, para obter sucesso ao final do tratamento (Souza, 2015).

A complexidade anatômica do SCR, é outro aspecto que pode implicar no sucesso do tratamento, na qual, durante a limpeza e desinfecção dos canais, a ação mecânica por si só não consegue realizar a instrumentação apropriada, dessa forma, torna-se necessário incorporar uma irrigação a este preparo, empregando agentes químicos apropriados, o que intensificará a assepsia do SCR (Rahimi et al., 2014). 
A utilização das soluções irrigadoras é essencial no PQM, contribuindo para limpeza do SCR, na remoção de conteúdos sépticos e na dissolução dos restos de tecidos necróticos da polpa, favorecendo assim a restauração dos tecidos perirradiculares (Lopes \& Siqueira, 2013).

Para que uma solução irrigante tenha sua ação ao longo do preparo do SCR, é necessário que apresente as seguintes propriedades, sendo os principais: ação antimicrobiana, dissolução tecidual, ser biocompatível aos tecidos periapicais, quantidade necessária, a concentração e o tempo de ação para que a solução seja capaz de apresentar o efeito esperado. Ademais, deve agir como lubrificantes, favorecendo a movimentação dos instrumentos no interior do canal radicular, modificar o pH do meio e evitar o escurecimento da superfície dentária (Haapasalo, Shen, Wang, \& Gao, 2014; Silva et al., 2016).

Dessa forma, a interpretação dos SCR requer do cirurgião-dentista planejamento pré-operatório adequado com avaliação clínica e exames complementares, como também habilidades táteis em relação as dificuldades que poderão aparecer ao decorrer do tratamento. Sendo assim, é necessário observar a anatomia interna dos canais os quais serão tratados, respeitando a morfologia do SCR, bem como a seleção do agente irrigante durante a execução da etapa operatória para que possa obter êxito no tratamento (Souza, 2015; Luz et al., 2019).

Em vista disso, muitas soluções irrigadoras foram propostas ao longo dos anos, dentre elas: o MTAD, que auxilia na eliminação efetiva do smear layer e Enterococcus faecalis que são resistentes a irrigantes endodônticos convencionais; o QMix, que é um novo irrigante endodôntico que promove a remoção com eficiência da camada de esfregaço; Smear Clear, é uma solução de EDTA a 17\% contendo um surfactante catiônico e um aniônico, sendo um agente eficaz na remoção da camada de esfregaço e na limpeza do canal radicular; e o peróxido de hidrogénio sendo outro desinfetante que há muito tempo é utilizado como solução de irrigação em endodontia. É eficaz contra bactérias, vírus e leveduras, mas sua eficácia antibacteriana é considerada fraca. No entanto, o NaOCl, o EDTA e a CHX continua sendo as substancias químicas mais utilizadas da terapia endodôntica (Dhawan et al., 2019; Mirhadi et al., 2015; Yadav, Yadav, Chandra, \& Tikku, 2017).

\subsection{Hipoclorito de Sódio $(\mathrm{NaOCl})$}

$\mathrm{O} \mathrm{NaOCl}$ é uma substância química, que faz parte do grupo dos halogenados, e a sua utilização iniciou no final do século XVIII, em 1792, e na atualidade é o composto químico mais empregado para realização do PQM dos canais radiculares. Foi inserido na endodontia por Blass, adotado por Walker (1936) e divulgado por Grossman (Leonardo \& Tanomaru, 2017).

Durante décadas o $\mathrm{NaOCl}$ têm sido a substância irrigante mais empregada por demostrar ação antimicrobiana e dissolver tecido orgânico, proporcionando o sucesso no tratamento do SCR. Alguns pesquisadores estudaram diversas concentrações do $\mathrm{NaOCl}$ e suas ações, e concluíram que as mais indicadas para a aplicação na terapia endodôntica são $0.5 \%$, $1.0 \%, 2.5 \%$ e $5.25 \%$. Contudo, a concentração ideal para irrigação em endodontia, ainda não foi formulada, mas o uso da solução em 2,5\% apresenta uma maior eficácia na eliminação de bactérias, expondo um equilíbrio das implicações causadas por concentrações elevadas (Roças, Provenzano, Neves, \& Siqueira, 2016; Ruksakiet et al., 2020).

De acordo com Lopes \& Siqueira (2013) o $\mathrm{NaOCl}$ pode ser identificado em uma série de produtos contendo concentrações e aditivos variáveis, tais como: solução de Dakin 0,5\%, neutralizada por ácido bórico para reduzir o pH $(\mathrm{pH}$ próximo de neutro); líquido de Dausfrene 0,5\%, neutralizada por bicarbonato de sódio; solução de Milton1,0\%, estabilizada por cloreto de sódio (16\%); água sanitária 2 a 2,5\%; solução de Labarraque 2,5\% e a soda clorada 4,0 e 6,0 \%.

$\mathrm{O} \mathrm{NaOCl}$, exibe excelentes propriedades, como potente ação antimicrobiana, capacidade de dissolver material orgânico, efeito lubrificante, desodorizante, clareador, apresenta baixa tensão superficial, tem um baixo custo e expõe um bom tempo de meia vida (Silva et al., 2015). 
Em virtude das suas características físicas-químicas, o $\mathrm{NaOCl}$ é um composto químico não quelante, por essa razão a sua utilização é apropriada em cada etapa do PQM de dentes necrosados ou com vitalidade, sua ação bactericida fundamentase no cloro ativo que é dispensado (Iandolo, Dagna, \& Abdellatif, 2019).

Embora, o $\mathrm{NaOCl}$ seja considerado a melhor solução irrigadora para a endodontia, existe relatos de processos alérgicos resultante de sua utilização, que pode induzir uma reação inflamatória local provocando dor severa (SIQUEIRA et al., 2014).

Entre as desvantagens do $\mathrm{NaOCl}$, estão a citotoxicidade nos tecidos periapicais, podendo causar irritação, cheiro e gosto desagradáveis e provocar manchamento de vestimentas. Além disso, altera a rigidez do dente pós terapia endodôntica o que pode encaminhar para o aumento de fraturas. O seu armazenamento requer maior cuidado, pois são soluções muito instáveis fazendo com que percam seu teor de cloro ativo, podendo ser influenciado por fatores locais tais como exposição à luz solar ou a temperaturas elevadas, devendo ser mantido em recipientes escuros e, se possível, refrigerados (Luz et al., 2019; Silva et al., 2016).

Estudos mostram que esta solução não consegue dissolver partículas inorgânicas e impedir a produção de smear layer que é um substrato amorfo, irregular e granuloso, que provém diretamente do preparo mecânico das paredes do canal radicular, passando a ser formado em decorrência natural da movimentação dos instrumentos cortantes no interior do canal em meio umedecido. Essa lama endodôntica é composta por raspas de dentina, material orgânico decorrente da decomposição tecidual e microrganismo, que aderem a parede do canal e obstruem a abertura dos túbulos dentinários, sendo capaz de impedir o alcance ao SCR e, consequentemente a ação de agentes antimicrobianos (Dewi, Upara, Chaiariyakul, \& Louwakul, 2020; Orlowski et al., 2020; Rajakumaran, Ramesh, Ashok, Balaji, \& Ganesh, 2019).

Na terapia de canais infectados há uma grande razão para a eliminação da lama dentinária. Nessas circunstâncias, as bactérias penetram nos túbulos dentinários podendo ser encontradas mais profundamente na dentina. Mesmo após o PQM do canal, algumas bactérias permanecem. Na falta do cemento radicular e na presença de comunicação com o exterior, as bactérias presentes podem iniciar e/ou manter complicações, como reabsorção radiculares e patologias perirradiculares, no entanto, a presença da lama dentinária ampara as bactérias, impedindo o contato dos medicamentos nas paredes do canal, ou até mesmo, que penetrem nós túbulos dentinários (Lopes \& Siqueira, 2013).

\subsection{Clorexidina (CHX)}

A CHX é uma substância antimicrobiana de amplo espectro, ativa contra bactérias gram-negativas e gram-positivas. Surgiu no final da década de 40, mas só em 1959 manifestou-se na odontologia como substância segura, a ser aplicada no controle do biofilme dental. Tem como finalidades na endodontia, ser um agente antimicrobiano no decorrer das etapas de irrigação e instrumentação do SCR, desinfeção do campo operatório, erradicação dos tecidos necróticos, PQM antes da desobstrução, medicação intracanal, desinfeção e remoção dos cones de guta percha nos retratamentos (Gomes et al., 2013; Sarmento, Guimaraes, \& Gomes, 2020).

Esta substância vem sendo empregada como solução irrigante e medicação intracanal em virtude da sua ação antibacteriana de amplo espectro, baixa citotoxicidade e por apresentar substantividade, pois, ela se liga à hidroxiapatita do esmalte ou dentina e a grupos aniônicos ácidos de glicoproteínas, sendo lentamente liberada à medida que a sua concentração no meio decresce, possibilitando desse modo um tempo de atuação prolongado (Lopes \& Siqueira, 2013).

Pode ser identificado na forma líquida ou em gel, cujas concentrações variam entre 0,2 e $2 \%$. A CHX em gel a $2 \%$ apresenta vantagens em relação à solução de $2 \%$ líquida, embora ambas tenham biocompatibilidade e ação antimicrobiana semelhantes. A CHX em gel lubrifica as paredes do canal radicular, diminuindo a fricção entre o instrumento e a superfície da 
dentina, favorecendo a instrumentação, além de diminuir o risco de fratura do instrumento dentro do canal (Gomes et al., 2013; Silveira et al., 2019).

A CHX é o agente irrigante selecionado quando há relato, por parte do paciente, de alergia/hipersensibilidade ao $\mathrm{NaOCl}$. Esta, é indicada no tratamento dos elementos dentários com polpa necrosada relacionada à rizogênese incompleta, onde nota-se grande risco de extravasamento apical da solução química, nos casos em que os microrganismos são resistentes ao TE e nas lesões refratárias (Bottcher, Sehnem, Montagner, Parolo, \& Grecca, 2015).

Esta substância pode ser empregada como solução de escolha, somente quando o único requisito é a ação antimicrobiana, visto que, esta solução embora seja tão efetiva, sob o espectro de atividade antimicrobiana, quanto o $\mathrm{NaOCl}$, não apresenta capacidade para dissolver tecido pulpar (Ruksakiet et al., 2020).

No entanto, a CHX demonstra algumas implicações que estão associadas a pigmentação da superfície dentária, deixando-a com uma coloração amarronzada. Outras desvantagens é que contém aroma desagradável, pode afetar o paladar, deixando com gosto metálico, descamação da mucosa, apresentar resposta alérgica e em alta concentração pode causar irritação na conjuntiva ocular e na pele (Gomes et al., 2013; Marion, Pavan, Arruda, Nakashima, \& Moraes, 2013).

Em contrapartida, ao contrário do $\mathrm{NaOCl}$, a $\mathrm{CHX}$ é incapaz de realizar a dissolução tecidual. Em conjunto têm sido indicados para elevar as propriedades das duas substâncias. A sequência de irrigação usada com os dois, foi proposto, mas identificou-se a produção de um precipitado denso e de coloração amarronzada, que pode afetar a estética do dente, pela difícil remoção (Agrawal, Mahant, Kapoor, \& Patel, 2014; Zandi et al., 2016).

\subsection{Reação entre a Clorexidina (CHX) e o Hipoclorito de Sódio ( $\mathrm{NaOCl})$}

$\mathrm{O} \mathrm{NaOCl}$ apresenta características importantes como a ação antimicrobiana e o potencial de dissolução de tecidos. Em altas concentrações o $\mathrm{NaOCl}$ expõe uma maior efetividade comparado a CHX, em contrapartida, podem provocar prejuízos aos tecidos perirradiculares (Silva et al., 2016; Ulusoy, Savur, Alaçam, \& Çelik, 2018).

Em comparação ao $\mathrm{NaOCl}$, a CHX apresenta excelentes resultados quando empregada como substância no tratamento do SRC. Vem se mostrando como possível substituto do $\mathrm{NaOCl}$ por resultar uma menor toxicidade, ter ação antimicrobiana contra espécies resistentes ao $\mathrm{NaOCl}$, menor tensão superficial e por demonstrar ação residual após a interação com a dentina, fenômeno conhecido como substantividade. No entanto, a CHX não apresenta um potencial de degradação dos tecidos orgânicos, uma importante condição das soluções químicas que são usadas ao longo da preparação dos canais, sendo associada com o $\mathrm{NaOCl}$ por preencherem as condições de uma solução irrigante ideal na endodontia (EcheverrI \& Alderete, 2015).

De acordo Chubb (2019), a relação entre a $\mathrm{CHX}$ e o $\mathrm{NaOCl}$ promove a formação instantânea de um precipitado de coloração acastanhada. Observando o aparecimento de íons de Cálcio, Magnésio e Ferro por meio da espectrometria de absorção atômica, que evidenciou uma substância carcinogênica e tóxica, denominada paracloroanilina (PCA). Esse precipitado, além de estimular uma lesão carcinogênica pode causar danificações ao periápice, favorecendo o manchamento na dentina e impossibilitando a obturação tridimensional do SCR (Mohammadi, Giardino, \& Asgary, 2015).

Pesquisas, ainda relatam que quando a reação ocorre dentro do canal, pode trazer complicações para a obturação, do mesmo modo para a permeabilidade dentinária, resultados que estão referentes à obliteração da rede de canais adventícios e dos túbulos dentinários pelo precipitado, que teria capacidade de se aderir à parede interna do canal, semelhante a um smear layer químico (Agrawal et al., 2014).

\section{5 Ácido Etilenodiamino Tetra-Acético (EDTA)}

O EDTA foi adotado na endodontia no final dos anos 50, para auxiliar a instrumentação de canais radiculares de menor diâmetro. É uma substância quelante com propriedades lubrificantes e amplamente utilizado. Essa substância química, 
remove os detritos inorgânicos e agem inibindo os íons metálicos como o cobre, ferro e magnésio. Este agente foi empregado no TE por ser benéfico na eliminação do smear layer preparando o canal para a obturação (Darcey, Jawad, Taylor, Roudsari, \& Hunter, 2017; Mafra et al., 2017).

O EDTA permeabiliza os canais atrésicos e elimina a smear layer das suas paredes. É um composto tensoativo que auxilia na redução da tensão superficial, melhorando a circulação e penetração no interior do canal. A solução aquosa de EDTA ideal é a de $17 \%$. O modo de utilização compreende em localizar a embocadura do canal radicular, preenchendo-o com EDTA durante um minuto, aprimorando assim a sua preparação químico-mecânica e aumentando a amplitude dos canais atresiados (Mafra et al., 2017).

Com relação ao processo de utilização do EDTA, a aplicação combinada de $\mathrm{NaOCl}$ e o EDTA foi indicado para remover os componentes orgânicos e inorgânicos do smear layer de forma eficaz. Dessa forma, para a desinfecção dos SCR é importante que haja um protocolo de irrigação final que ocorra de forma eficiente, preenchendo o canal radicular com EDTA, com posterior irrigação com NaOCl, para melhorar o PQM e eliminar o smear layer (Guo et al., 2014; Mafra et al., 2017).

Uma desvantagem da combinação entre $\mathrm{NaOCl}$ e o EDTA, é a ação potencializada, que ocorre sobre a dentina, na qual resulta na degradação esporádica das paredes dos canais, o que possibilita à dissolução da dentina inter e peritubular, causando a desgaste das propriedades mecânicas da dentina. A perda da mineralização ocorre em razão do EDTA, por não ter padrão constante ao longo dos túbulos dentinários, desta forma, a remoção do componente orgânico pelo $\mathrm{NaOCl}$ aumenta a permeabilidade ao EDTA, que vai dissolver a hidroxiapatita, expondo as fibras de colágeno e acelerando o processo infiltrativo do $\mathrm{NaOCl}$ (Baldasso, Roleto, Silva, Morgental, \& kopper, 2017; Mafra et al., 2017).

\section{Resultados}

O presente trabalho avaliou por meio de uma revisão de literatura, as propriedades, vantagens e desvantagens das soluções irrigadoras mais utilizadas no preparo químico-mecânico dos canais radiculares.

A princípio, foram realizadas coletas dos dados contendo as informações dos artigos a respeito das soluções irrigadoras, por meio do levantamento bibliográfico que estarão apresentadas na Tabela 1. Seguindo os critérios de inclusão, foram identificadas no Scielo um total de 56 referências bibliográficas, sendo selecionado 3 artigos. Já no Lilacs foram identificadas 299 referências e considerados 5 artigos. No PubMed, foram identificadas 161 referências, permanecendo 7 artigos. Foram encontrados 136 artigos na Science Direct, sendo selecionado 5. Desta forma, a partir da análise final, 20 artigos fizeram parte do presente estudo.

Tabela 1. Resultados do levantamento bibliográfico.

\begin{tabular}{|l|l|l|}
\hline AUTOR/ANO & TÍTULO & RESULTADOS \\
\hline DARDA et al., 2014. & $\begin{array}{l}\text { An in-vitro evaluation of effect of edtac on } \\
\text { root dentin with respect to time. }\end{array}$ & $\begin{array}{l}\text { Os resultados mostraram que 1 min de irrigação com EDTA é } \\
\text { eficaz na remoção da camada de esfregaço. No entanto, uma } \\
\text { aplicação de 10 minutos de EDTA causou erosão dentinária } \\
\text { peritubular e intertubular excessiva }\end{array}$ \\
\hline $\begin{array}{l}\text { HAAPASALO et al., } \\
2014 .\end{array}$ & $\begin{array}{l}\text { Irrigation in endodontics. } \\
\text { dissolver matéria orgânica, em alta concentração tem melhor } \\
\text { efeito em relação as soluçães de 1 e 2\%; } \\
\text { O EDTA é necessário com enxague final para remoção de } \\
\text { smear layer. }\end{array}$ \\
\hline MARINHO et al., 2014. & $\begin{array}{l}\text { Monitoring the effectiveness of root canal } \\
\text { procedures on endotoxin levels found in } \\
\text { teeth with chronic apical periodontitis. }\end{array}$ & $\begin{array}{l}\text { Houve redução mediana de endotoxinas, independente do } \\
\text { irrigante. Irrigação final com EDTA reduziu discretamente os } \\
\text { níveis de endotoxina quando usado após CHX. }\end{array}$ \\
\hline $\begin{array}{l}\text { ECHEVERRI; } \\
\text { ALDERETE, 2015. }\end{array}$ & $\begin{array}{l}\text { In vitro Antibacterial Effect of 2\% } \\
\text { Chlorhexidine Against Enterococcus }\end{array}$ & $\begin{array}{l}\text { Foi observado que o NaOCl 5\% + H2O+CHX 2\%, apresentou } \\
\text { maior inibição dos microrganismos. }\end{array}$ \\
\hline
\end{tabular}




\begin{tabular}{|c|c|c|}
\hline & $\begin{array}{l}\text { faecalis in Dentin Previously Irrigated with } \\
5 \% \text { Sodium Hypochlorite. }\end{array}$ & \\
\hline MA et al., 2015. & $\begin{array}{l}\text { The effects of sodium hypochlorite and } \\
\text { chlorhexidine irrigants on the antibacterial } \\
\text { activities of alkaline media against } \\
\text { Enterococcus faecalis. }\end{array}$ & $\begin{array}{l}\text { Um minuto de pré-tratamento com } 2 \% \text { de } \mathrm{CHX}, 0,2 \% \text { de } \mathrm{CHX} \\
\text { ou } 5,25 \% \text { de } \mathrm{NaOCl} \text { em combinação com um desafio alcalino } \\
\text { subsequente diminuiu significativamente as taxas de } \\
\text { sobrevivência de } E \text {. faecalis planctônica, mas o pré-tratamento } \\
\text { com } 1 \% \text { de } \mathrm{NaOCl} \text { não. }\end{array}$ \\
\hline $\begin{array}{l}\text { MOHAMMADI et al., } \\
2015 .\end{array}$ & $\begin{array}{l}\text { Agonistic and Antagonistic Interactions } \\
\text { between Chlorhexidine and Other } \\
\text { Endodontic Agents: A Critical Review. }\end{array}$ & $\begin{array}{l}\text { Mostraram que a combinação da } \mathrm{CHX} \text { e o } \mathrm{NaOCl} \text { causam } \\
\text { mudança de cor e a formação de um precipitado neutro e } \\
\text { insolúvel; } \\
\text { A combinação da CHX com o EDTA forma um sal; }\end{array}$ \\
\hline SEELAN et al., 2015. & $\begin{array}{l}\text { Comparative evaluation of effect of } \\
\text { different irrigation solutions against } \\
\text { Enterococcus faecalis: A polymerase chain } \\
\text { reaction-based study. }\end{array}$ & $\begin{array}{l}\text { Os resultados mostraram que o } \mathrm{NaOCl} \text { a } 5 \% \text { seguido de EDTA } \\
\text { a } 17 \% \text { e } \mathrm{CHX} \text { a } 2 \% \text {, apresentou atividade antimicrobiana } \\
\text { máxima em todos os três intervalos de tempo diferentes. }\end{array}$ \\
\hline $\begin{array}{l}\text { GONÇALVES et al., } \\
2016 .\end{array}$ & $\begin{array}{l}\text { The effect of sodium hypochlorite and } \\
\text { chlorhexidine as irrigant solutions for root } \\
\text { canal disinfection: a systematic review of } \\
\text { clinical trials. }\end{array}$ & $\begin{array}{l}\text { O NaOCl } 2,5 \% \text { foi mais eficaz do que a } \mathrm{CHX} 2 \% \text { na redução } \\
\text { de microrganismo em um estudo, e outro relatou resultados } \\
\text { opostos. Ambos as soluções irrigadoras foram ineficazes na } \\
\text { eliminação de toxinas. }\end{array}$ \\
\hline SILVA et al., 2016. & $\begin{array}{l}\text { Antimicrobial activity irrigating solutions } \\
\text { to prepare channels biomechanical root face } \\
\text { to Enterococcus Faecalis. }\end{array}$ & $\begin{array}{l}\mathrm{O} \mathrm{NaOCl} 2,5 \% \text { foi a solução que apresentou melhor atividade } \\
\text { antimicrobiana, seguida da CHX } 2 \% \text {. }\end{array}$ \\
\hline ROÇAS et al., 2016. & $\begin{array}{l}\text { Disinfecting effects of rotary } \\
\text { instrumentation with either } 2.5 \% \text { sodium } \\
\text { hypochlorite or } 2 \% \text { chlorhexidine as the } \\
\text { main irrigant: a randomized clinical study. }\end{array}$ & $\begin{array}{l}\text { Tanto o } \mathrm{NaOCl} 2,5 \% \text { quanto o } \mathrm{CHX} 2 \% \text {, foram eficazes na } \\
\text { redução dos microorganismos. }\end{array}$ \\
\hline ZANDI et al., 2016. & $\begin{array}{l}\text { Antibacterial effectiveness of } 2 \text { roots canal } \\
\text { irrigants in root-filled teeth with infection: a } \\
\text { randomized clinical trial. }\end{array}$ & $\begin{array}{l}\text { Nenhuma diferença significativa entre } \mathrm{NaOCl} 1 \% \text { e } \mathrm{CHX} 2 \% \\
\text { foi encontrado. }\end{array}$ \\
\hline ALEGRE et al., 2017. & $\begin{array}{l}\text { Determination of residual parachloaniline } \\
\text { produced by endodontic treatment after the } \\
\text { use of } 5 \% \text { sodium hypochlorite and } 2 \% \\
\text { chlorhexidine combined: na ex-vivo study. }\end{array}$ & $\begin{array}{l}\text { A formação de PCA ocorreu na presença de } 2 \% \text { de CHX e } 5 \% \\
\text { de NaOCl. }\end{array}$ \\
\hline BERALDO et al., 2017. & $\begin{array}{l}\text { Scanning electron microscopic evaluation } \\
\text { of smear layer removal using isolated or } \\
\text { interweaving EDTA with sodium } \\
\text { hypochlorite. }\end{array}$ & $\begin{array}{l}\text { O uso alternado de EDTA } 17 \% \text { durante a instrumentação com } \\
\mathrm{NaOCl} 2,5 \% \text { foi o método de irrigação mais eficaz para } \\
\text { remover a camada de esfregaço apical nos terços coronário e } \\
\text { médio dos canais. }\end{array}$ \\
\hline $\begin{array}{l}\text { BUKHARY; BALTO, } \\
2017 .\end{array}$ & $\begin{array}{l}\text { Antibacterial Efficacy of Octenisept, } \\
\text { Alexidine, Chlorhexidine, and Sodium } \\
\text { Hypochlorite against Enterococcus faecalis } \\
\text { Biofilms. }\end{array}$ & $\begin{array}{l}\text { A maior proporção de células mortas foi encontrada no grupo } \\
\text { de } \mathrm{NaOCl} 5,25 \% \text { em comparação a CHX } 2 \% \text {. }\end{array}$ \\
\hline MAFRA et al., 2017. & $\begin{array}{l}\text { The effectiveness of EDTA solution in } \\
\text { removing smear layer and its relation to the } \\
\text { time of use: an integrative review. }\end{array}$ & $\begin{array}{l}\text { Verificou-se que o tempo de uso do EDTA interferiu } \\
\text { diretamente na qualidade da remoção da smear layer. Além } \\
\text { disso, verificou-se que o EDTA a } 17 \% \text { utilizado por um } \\
\text { período de um minuto foi ideal para a remoção da smear layer, } \\
\text { enquanto que a aplicação de EDTA no tempo superior a sete } \\
\text { minutos provocou erosão da dentina peritubular. }\end{array}$ \\
\hline NOCCA et al., 2017. & $\begin{array}{l}\text { Chromographic Analysis and Cytotoxic } \\
\text { Effects of Chlorhexidine and Sodium } \\
\text { Hypochlorite Reaction Mixtures. }\end{array}$ & $\begin{array}{l}\text { Mostraram que a associação do } \mathrm{NaOCl} \text { com a } \mathrm{CHX} \text {, exibe uma } \\
\text { ampla gama de efeitos citotóxicos. }\end{array}$ \\
\hline SERRANO et al., 2018. & $\begin{array}{l}\text { Influência de la activación ultrasónica del } \\
\text { hipoclorito de sodio y de la clorhexidina } \\
\text { sobre el biofilm: estudio ex vivo. }\end{array}$ & $\begin{array}{l}\text { O hipoclorito de sódio a } 5 \% \text { é mais eficiente do que o } \\
\text { clorexidina a } 2 \% \text { na remoção do biofilme, pois deixa o terço } \\
\text { médio mais limpo. Ambas as soluções apresentam } \\
\text { comportamento semelhante no terço cervical das amostras. }\end{array}$ \\
\hline $\begin{array}{l}\text { NEELAKANTAN } \quad e t \\
a l ., 2019 .\end{array}$ & $\begin{array}{l}\text { Endotoxin levels after chemomechanical } \\
\text { preparation of root canals with sodium } \\
\text { hypochlorite or chlorhexidine: a systematic } \\
\text { review of clinical trials and meta-analysis. }\end{array}$ & $\begin{array}{l}\text { O uso de } \mathrm{NaOCl} \text { e } \mathrm{CHX} \text { durante o preparo quimio-mecânico } \\
\text { reduziu significativamente os níveis de LPS; } \\
\text { Quando o NaOCl foi usado durante o preparo quimio- } \\
\text { mecânico, os níveis de endotoxinas foram menores do que } \\
\text { aqueles obtidos após o preparo quimio-mecânico com CHX. }\end{array}$ \\
\hline
\end{tabular}




\begin{tabular}{|l|l|l|}
\hline $\begin{array}{l}\text { RUKSAKIET et al., } \\
2020 .\end{array}$ & $\begin{array}{l}\text { Antimicrobial efficacy of chlorhexidine and } \\
\text { sodium hypochlorite in root canal } \\
\text { disinfection: a systematic review and meta- } \\
\text { analysis of randomized controlled trials. }\end{array}$ & $\begin{array}{l}\text { Tanto CHX 2\% quanto NaOCl 2,5\%, reduziram as infecções } \\
\text { bacterianas após irrigação, sem qualquer diferença significativa } \\
\text { na eficácia antimicrobiana entre eles. }\end{array}$ \\
\hline CORTÉS et al., 2020. & $\begin{array}{l}\text { Capacidad de Disolución del Hipoclorito de } \\
\text { Sodio con o sin Activación. }\end{array}$ & $\begin{array}{l}\text { NaOCl } 1 \% \text { tem uma capacidade de dissolução inferior que é } \\
\text { ligeiramente melhorada quando ativado. NaOCl 3\% tem } \\
\text { melhor capacidade de dissolução do que NaOCl } 1 \% \text { mas a } \\
\text { ativação não melhora significativamente. O NaOCl 5\% possui } \\
\text { maior capacidade de dissolução, que aumenta com a ativação, } \\
\text { sem significância entre a ativação sônica e ultrassônica. }\end{array}$ \\
\hline
\end{tabular}

Fonte: Autores (2021).

\section{Discussão}

De acordo os resultados presentes na Tabela 1, o estudo realizado por Zandi et al., (2016) relataram a comparação dos efeitos antimicrobianos, e observaram que $\mathrm{NaOCl} 1 \%$ e a $\mathrm{CHX} 2 \%$ não apresentam diferenças significativas em seus efeitos, pois ambas reduziram a contagem de bactérias dos canais radiculares. Já nos estudos de Gonçalves et al., (2016); Silva et al., (2016), o uso do $\mathrm{NaOCl}$ 2,5\% como solução irrigadora apresentou maior redução de microrganismos ao ser comparada com a CHX 2\%. No entanto, Roças et al., (2016); Ruksakiet et al., (2020) observaram que tanto a CHX 2\% quanto o $\mathrm{NaOCl} 2,5 \%$ apresentaram eficácia antimicrobiana sem qualquer diferença.

Analisando os efeitos do $\mathrm{NaOCl}$, Gonçalves et al., (2016); Silva et al., (2016), concluíram que esta solução tem sido a substância mais utilizada no preparo dos SCR, devido a sua atividade antimicrobiana e sua capacidade de dissolução de tecidos orgânicos. Os resultados de seus estudos demostraram que o $\mathrm{NaOCl}$ 2,5\% apresentou maior efetividade comparado a CHX 2\% na eliminação dos microrganismos. No entanto, no mesmo estudo, Gonçalves et al., (2016) relataram resultados opostos. Ambos as soluções irrigadoras foram ineficazes na eliminação de toxinas.

Cortés, Uribe, Arriagada e Reveco (2020) em seu estudo, observaram o $\mathrm{NaOCl}$ em diversas concentrações antes e após ativação, e verificaram que o $\mathrm{NaOCl} 1 \%$ possui um menor efeito na dissolução de tecido orgânico, melhorando após sua ativação. Para o $\mathrm{NaOCl}$ a 3\% teve uma melhora ao ser comparado com 1\% de $\mathrm{NaOCl}$ seguido da ativação, porém o $\mathrm{NaOCl} 5 \%$ foi o que apresentou maior capacidade de dissolução de tecido orgânico quando a ativação é realizada.

No estudo realizado por Ma, Tong, Ling, Liu e Wei (2015), demostraram diferentes protocolos de irrigação com $\mathrm{NaOCl}$ e CHX em diversas concentrações, e concluíram que 1 minuto de pré-tratamento com 2\% de CHX, 0,2\% de CHX ou $5,25 \% \mathrm{NaOCl}$ diminuiu significativamente as taxas de sobrevivência de Enterococcus faecalis, porém o pré-tratamento com $1 \%$ de $\mathrm{NaOCl}$ não houve redução dos microrganismos.

Echeverri e Alderete (2015), no seu estudo, analisaram o efeito antibacteriano da CHX 2\% contra Enterococcus faecalis na dentina, preparando diferentes protocolos de irrigação. Assim, observaram que o uso do $\mathrm{NaOCl}$ a $5 \%$ e $\mathrm{H} 2 \mathrm{O}$ antecedido da CHX a 2\%, resultou em uma maior inibição dos microrganismos. Contudo, Seelan et al., (2015) em seu trabalho, observaram que a ação do $\mathrm{NaOCl}$ a $5 \%$ seguido de EDTA a $17 \%$ e $\mathrm{CHX}$ a $2 \%$, resultou em uma máxima atividade antimicrobiana.

Estudo feito por Haapasalo et al., (2014), mostraram que o $\mathrm{NaOCl} \mathrm{em} \mathrm{altas} \mathrm{concentrações} \mathrm{apresenta} \mathrm{melhor} \mathrm{efeito} \mathrm{em}$ relação as soluções de 1 e $2 \%$ seguida da utilização do EDTA como enxaguante final para a remoção do smear layer. Serrano et al. (2018), avaliaram em seu estudo que o uso do $\mathrm{NaOCl}$ a $5 \%$ é mais eficiente do que o clorexidina a $2 \%$ na remoção do biofilme, realizando melhor desinfecção do terço médio. Em outra pesquisa semelhante realizada por Bukhary e Balto (2017), mostrou que o uso do $\mathrm{NaOCl}$ a $5,25 \%$ provocou uma maior proporção de células mortas ao comparar com a CHX a $2 \%$ por apresentar uma maior concentração.

O uso alternado de EDTA 17\% durante a instrumentação com $\mathrm{NaOCl}$ 2,5\% foi o método de irrigação mais eficaz para 
remover a camada de esfregaço apical nos terços coronário e médio dos canais, segundo o estudo de Beraldo, Silva e Nunes (2017). Em outra pesquisa, realizado por Marinho, Martinho, Zaia e Ferraz (2014) observaram que a utilização do EDTA na irrigação final após a CHX reduziu discretamente os níveis de endotoxina.

Já Darda et al., (2014) mostraram em seus resultados que 1 minuto de irrigação com EDTA é eficaz na remoção da camada de esfregaço. No entanto, uma aplicação de 10 minutos de EDTA causou erosão dentinária peritubular e intertubular excessiva. Em concordância, Mafra et al., (2017) também verificaram o tempo de uso do EDTA 17\% na remoção do smear layer, concluindo que o intervalo de 1 minuto foi ideal, porém, o tempo superior a 7 minutos provocou erosão da dentina peritubular.

Mohammadi et al (2015); Nocca et al., (2017), ressaltam em seus estudos que a combinação do $\mathrm{NaOCl}$ com a CHX causa efeitos citotóxicos, ocorre mudança de cor e formação de um precipitado neutro insolúvel. Mohammadi et al., (2015), ainda relata que ao associar a CHX com EDTA ocorre a formação de um sal. Em concordância, no estudo feito por Alegre et al., (2017), comprovaram que a combinação do $\mathrm{NaOCl} 5 \%$ com a CHX 2\% ocorreu a formação de um precipitado denominado Paracloroanilina (PCA), apresentando citotoxicidade e um alto potencial carcinogênico em humanos. Dessa forma, relataram a necessidade de um protocolo de irrigação para reduzir possíveis contaminações.

\section{Considerações Finais}

As substancias irrigadoras apresentadas são as mais empregadas na endodontia por apresentarem propriedades e ações que permitem a eliminação dos microrganismos e na remoção do smear layer presente no interior do canal infectado. A irrigação com essas substâncias é uma etapa fundamental para a desinfecção do canal radicular, assegurando a assepsia e favorecendo no prognóstico.

Em virtude dos aspectos presentes no trabalho, foi possível observar as características das soluções irrigadoras e suas combinações, bem como suas vantagens e desvantagens. A partir disso, percebe-se a necessidade do conhecimento por parte do cirurgião-dentista acerca das soluções que são utilizadas para o tratamento como também a suas aplicações e propriedades, com o propósito de compreender os resultados que serão alcançados na etapa operatória de irrigação.

Dessa forma, destaca-se a importância de mais estudos, pesquisas e publicações de trabalhos abordando as substâncias irrigadoras no tratamento endodôntico na qual, os profissionais possam selecionar corretamente o agente químico conforme o tratamento, com o objetivo de proporcionar o sucesso na terapia endodôntica.

\section{Referências}

Agrawal, V. S., Mahant, R., Kapoor, S., \& Patel, M. (2014). A contemporary overview of endodontic irrigants-A review. Journal Dental Applications, 1 (6), 105-115. https://www.researchgate.net/profile/Rajesh-Mahant/publication/277721660_A_Contemporary_Overview_of_Endodontic_Irrigants_-_A_Review/li nks/5572810608aeb6d8c0175fff/A-Contemporary-Overview-of-Endodontic-Irrigants-A-Review.pdf

Alegre, O., Yévenes, I., Parada, J., Maggiolo, S., \& Fernández, E. (2017). Determination of residual parachloaniline produced by endodontic treatment after the use of 5\% sodium hypochlorite and 2\% chlorhexidine combined: na ex-vivo study. Rev. Clín. Periodoncia implantol. Rehabil. Oral, 10(3) 145-148. https://pesquisa.bvsalud.org/portal/resource/pt/biblio-900296

Baldasso, F. E. R., Roleto, L., Silva, V. D., Morgental, R. D., \& Kopper, P. M. P. (2017). Effect of final irrigation protocols on microhardness reduction and erosion of root canal dentin. Brazilian Oral Research, 31(1). https://www.scielo.br/scielo.php?script=sci_arttext\&pid=S1806$83242017000100237 \& \operatorname{lng}=$ en $\&$ nrm=iso\&tlng=en

Beraldo, Â. J. S., Silva, R. V., \& Nunes, E. (2017). Scanning electron microscopic evaluation of smear layer removal using isolated or interweaving EDTA with sodium hypochlorite. Iranian endodontic journal, 12(1), 55-59. https://www.ncbi.nlm.nih.gov/pmc/articles/PMC5282380/

Bottcher, D. E., Sehnem, N. T., Montagner, F., Parolo, C. C. F., \& Grecca, F. S. (2015) Evaluation of the effect of Enterococcus faecalis biofilm on the 2\% chlorhexidine substantivity: an in vitro study. Journal of endodontics, https://www.sciencedirect.com/science/article/abs/pii/S0099239915003854

Bukhary, S., \& Balto, H. (2017). Eficácia antibacteriana de octenisept, alexidina, clorexidina e hipoclorito de sódio contra biofilmes de Enterococcus faecalis. Journal of Endodontics, 43(4), 643-647. https://www.sciencedirect.com/science/article/abs/pii/S0099239916306173 
Chubb, D. W. R. A. (2019). Review of the prognostic value of irrigation on root canal treatment success. Australian Endodontic Journal, 45(1), 5-11. https://onlinelibrary.wiley.com/doi/abs/10.1111/aej.12348

Cortes, H. M., Uribe, M. T. M., Arriagada, F. A., \& Reveco, J. A. (2019) Capacidad de Disolución del Hipoclorito de Sodio con o sin Activación. Avances en Odontoestomatologia, 35(3), 113-118. http://scielo.isciii.es/scielo.php?script=sci_arttext\&pid=S0213-12852019000300003\&lng=es\&nrm=iso

Darcey, J., Jawad, S., Taylor, C., Roudsari, R. V., \& Hunter, M. (2017). Modern endodontic principles part 4: irrigation. Atualização dental, 43(1), 20-33. https://www.magonlinelibrary.com/doi/abs/10.12968/denu.2016.43.1.20

Darda, S., Madria, K., Jamenis, R., Heda, A., Khanna, A., \& Sardar, L. (2014). An in-vitro evaluation of effect of EDTAC on root dentin with respect to time Journal of International Oral Health, 6(2), 22-27. https://www.ncbi.nlm.nih.gov/pmc/articles/PMC4037788/

Dewi A., Upara, C., Chaiariyakul, D., \& Louwakul, P. (2020). Smear Layer Removal from Root Canal Dentine and Antimicrobial Effect of Citric Acidmodified Chlorhexidine. European Endodontic Journal, 5(3), 257-263. https://www.ncbi.nlm.nih.gov/pmc/articles/PMC7881384/

Dhawan R., Gupta, A., Dhillon, J. S., Dhawan, S., Sharma, T., \& Batra, D. (2019). Effect of different irrigating solutions with surfactants on the microhardness and smear layer removal of root canal dentin: An in vitro study. J Conserv Dent. 22(5), 454-458. https://www.ncbi.nlm.nih.gov/pmc/articles/PMC7537743/

Dioguardi, M., Gioia, G. D., Illuzzi, G., Laneve, E., Cocco, A., \& Troiano, G. (2018). Endodontic irrigants: Different methods to improve efficacy and related problems. European Journal of Dentistry, 12(3), 459-466. https://www.ncbi.nlm.nih.gov/pmc/articles/PMC6089055/

Echeverri, D. \& Alderete, D. (2015). In vitro Antibacterial Effect of 2\% Chlorhexidine Against Enterococcus faecalis in Dentin Previously Irrigated with 5\% Sodium Hypochlorite. Int. J. Odontostomat, 9(1), 25-29. https://scielo.conicyt.cl/scielo.php?script=sci_arttext\&pid=S0718$381 \mathrm{X} 2015000100004 \& \operatorname{lng}=\mathrm{es} \& \mathrm{nrm}=\mathrm{iso}$

Gomes, B. P. F. A., Vianna, M. E., Zaia, A. A., Almeida, J. F., Souza, F. J. F., \& Ferraz, C. C. (2013). Chlorhexidine in endodontics. Brazilian dental journal, 24(2), 89-102. https://pubmed.ncbi.nlm.nih.gov/23780357/

Gonçalves, L. S., Rodrigues, R. C. V., Andrade, C. V. Jr., Soares, R. G., \& Vettore, M. V. (2016). The effect of sodium hypochlorite and chlorhexidine as irrigant solutions for root canal disinfection: a systematic review of clinical trials. Journal of endodontics, 42(4), 527-532. https://www.jendodon.com/article/S0099-2399(15)01151-6/fulltext

Guo, X., Miao, H., Li, L., Zhang, S., Zhou, D., Lu, Y., \& Wu, L. (2014). Efficacy of four different irrigation techniques combined with 60 C 3\% sodium hypochlorite and 17\% EDTA in smear layer removal. BMC oral health, 14(1). https://bmcoralhealth.biomedcentral.com/articles/10.1186/1472-6831-14-114 Haapasalo, M., Shen, Y., Wang, Z., \& Gao, Y. (2019). Irrigation in endodontics. British dental journal, 216(6), 299-303. https://www.nature.com/articles/sj.bdj.2014.204.pdf

Iandolo, A., Dagna, A., \& Abdellatif, D. (2019). Evaluation of the actual chlorine concentration and the required time for pulp dissolution using different sodium hypochlorite irrigating solutions. J Conserv Dent. 22(2), 108-113. https://www.ncbi.nlm.nih.gov/pmc/articles/PMC6519176/

Leonardo, M. R., \& Tanomaru, J. M. G. (2017). Preparo biomecânico dos canais radiculares: definição, conceituação, importância, finalidades e recursos convencionais para sua aplicação. In: M. Leonardo, R \& R. Leonardo, T. Tratamento de canais radiculares (2a ed.), Cap. 4, pp. 25-37. Artes Médicas.

Lopes, H. P., \& Siqueira, J. F., Jr. (2013) Substâncias químicas empregadas no preparo dos canais radiculares. In: H. Lopes, P., \& J. Siqueira, F., Jr. Endodontia: biologia e técnica (3a ed.), Cap. 14, pp. 478-496. Guanabara KOOGAN

Luz, L. B., Santana, R. M. C., Prates, A. W., Frorhlich, J., Melo, T. A. F., Montagner, F., \& Luisi, S. B. (2019). Evaluation of smear layer formation in mechanical chemical preparation performed with $2.5 \%$ sodium hypochloride solution or gel. Journal of Health \& Biological Sciences, $7(2)$, 159-165. https://periodicos.unichristus.edu.br/jhbs/article/view/2328

Ma, J., Tong, Z., Ling, J., Liu, H., \& Wei, X. (2015). The effects of sodium hypochlorite and chlorhexidine irrigants on the antibacterial activities of alkaline media against Enterococcus faecalis. Archives of Oral Biology, 60, 1075-1081. https://www.sciencedirect.com/science/article/abs/pii/S0003996915000977

Mafra, S. C., Girelli, C. F. M., Xavier, V. F., Lacerda, M. F. L., Lacerda, G. P., \& Coelho, R. G. (2017). The effectiveness of EDTA solution in removing smear layer and its relationto the time of use: an integrative review. Revista da Faculdade de Odontologia-UPF, 22(1), 120-129. http://seer.upf.br/index.php/rfo/article/view/6305

Marinho, A. C. S., Martinho, F. C., Zaia, A. A., \& Ferraz, C. C. R. (2014). Monitoring the effectiveness of root canal procedures on endotoxin levels found in teeth with chronic apical periodontitis. Journal of applied oral science, 22(6), 490-495. https://www.scielo.br/scielo.php?pid=S1678$77572014000600490 \&$ script=sci_arttext

Marion, J., Pavan, K., Arruda, M. E. B. F., Nakashima, L., \& Morais, C. A. H. (2013). Chlorhexidine and its applications in Endodontics: a literature review. Dental Press Endod, 3(3). https://www.researchgate.net/profile/Jefferson-Marion/publication/287337747_Chlorhexidine_and_its_applicatio ns_in_ Endodontics_A_literature_review/links/56a76d4508ae0fd8b3fe024f/Chlorhexidine-and-its-applications-in-Endodontics-A-literature-review.pdf

Mirhadi, H., Abbaszadegan, A., Ranjbar, M. A., Azar, M. R., Geramizadeh, B., Torabi, S., Aleyasin, Z. S., \& Gholami, A. (2015). Efeito antibacteriano e tóxico do peróxido de hidrogênio combinado com diferentes concentrações de clorexidina em comparação com o hipoclorito de sódio. Journal of dentistry (Shiraz), 16 (4), 349-355. https://www.ncbi.nlm.nih.gov/pmc/articles/PMC4664033/

Mohammadi, Z., Giardino, L., \& Asgary, S. (2015). Agonistic and Antagonistic Interactions between Chlorhexidine and Other Endodontic Agents: A Critical Review. Iranian Endodontic Journal, 10(1), 1-5. https://www.ncbi.nlm.nih.gov/pmc/articles/PMC4293573/

Neelakantan, P., Herrera, D. R., Pecorari, V. G. A., \& Gomes, B. P. F. A. (2019). Endotoxin levels after chemomechanical preparation of root canals with sodium hypochlorite or chlorhexidine: a systematic review of clinical trials and meta-analysis. Int Endod J, 52 (1), 19-27. https://onlinelibrary.wiley.com/doi/full/10.1111/iej.12963 
Nocca, G., Ahmed, H. M. A., Martorana, G. E., Callà, C., Gambarini, G., Rengo, S., \& Spagnuolo, G. (2017). Chromographic Analysis and Cytotoxic Effects of Chlorhexidine and Sodium Hypochlorite Reaction Mixtures. Journal of endodontics, 43(9), 1545-1552. https://www.sciencedirect.com/science/article/abs/pii/S0099239917305319

Orlowski, N. B., Schimdt, T. F., Teixeira, C. S., Garcia, L. F. R., Savaris, J. M. Tay, F. R., \& Bortoluzzi, E. A. (2020). Smear layer removal using passive ultrasonic irrigation and diferente concentrations of sodium hypochlorite. Journal of endodontics, 46 (11), 1738-1744. https://www.sciencedirect.com/science/article/abs/pii/S0099239920305094

Ozkan, H. B., Cobankara, F. K., \& Ozer, F. (2020). Evaluation of the Antibacterial Effects of Single and Combined use of Different Irrigation Solutions Against Intracanal Enterococcus Faecalis. Acta Stomatol Croat. 54(3), 250-262. https://www.ncbi.nlm.nih.gov/pmc/articles/PMC7586900/

Rahimi, S., Jananib, M., Lotfic, M., Shahia, S. Aghbalia, A., Pakdeld, M. V., Milania, A. S., \& Ghasemib, N. (2014). A review of antibacterial agents in endodontic treatment. Iranian endodontic journal, 9 (3), 161-168. https://www.ncbi.nlm.nih.gov/pmc/articles/PMC4099945/pdf/iej-9-161.pdf

Rajakumaran, A., Ramesh, H., Ashok, R., Balaji, L., \& Ganesh A. (2019). Smear Layer Removal and Microhardness Alteration Potential of a Naturally Occurring Antioxidant - An In Vitro Study. Cureus, 25(11). https://www.ncbi.nlm.nih.gov/pmc/articles/PMC6759042/pdf/cureus-0011-00000005241.pdf

Rôças, I. N., Provenzano, J. C., Neves, M. A. S., \& Siqueira, J. F. Jr. (2016). Disinfecting effects of rotary instrumentation with either 2.5\% sodium hypochlorite or $2 \%$ chlorhexidine as the main irrigant: a randomized clinical study. Journal of endodontics, 42(6), 943-947. https://www.sciencedirect.com/science/article/abs/pii/S009923991630108X

Rother, E. T. (2007). Revisão sistemática X revisão narrativa. Acta paul. Enferm. 20 (2), 5-6. https://www.scielo.br/j/ape/a/z7zZ4Z4 GwYV6FR7S9FHTByr/?lang=pt

Ruksakiet, K., Hanák, N., Farkas, N., Hegyi, P., Sadaeng, W., Czumbel, L. M., Sang-Ngoen, T., Garami, A., Mikó, A., Varga, G., \& Lohinai, Z. (2020). Antimicrobial efficacy of chlorhexidine and sodium hypochlorite in root canal disinfection: a systematic review and meta-analysis of randomized controlled trials. Journal of endodontics, 46 (8), 1032-1041. https://www.sciencedirect.com/science/article/pii/S0099239920303083

Sarmento, E. B., Guimaraes, L. S., \& Gomes, C. C. (2020). The Influence of Sodium Hypochlorite and Chlorhexidine on Postoperative Pain in Necrotic Teeth: A Systematic Review. European Endodontic Journal, 5(3), 177-185. https://www.ncbi.nlm.nih.gov/pmc/articles/PMC7881388/

Seelan, R. G., Kumar, A., Jonathan, R., Maheswari, U., Raja, J., \& Chelliah, P. (2015). Comparative evaluation of effect of different irrigation solutions against Enterococcus faecalis: A polymerase chain reaction-based study. Journal of pharmacy \& bioallied sciences, 7(2). https://www.ncbi.nlm.nih.gov/pmc/articles/PMC4606663/

Serrano, N. D. M. (2018). Influência de la activación ultrasónica del hipoclorito de sodio y de la clorhexidina sobre el biofilm: Estudio ex vivo. Rev Cient Odontol, 6(2), 129-142. https://revistas.cientifica.edu.pe/index.php/odontologica/article/view/465/527

Silva, E. J. N L., Monteiro, M. R., Belladona, F. G., Almeida, J. F., De-Deus, G., \& Neves, A. A. (2015). Postoperative pain after foraminal instrumentation with a reciprocating system and different irrigating solutions. Brazilian dental journal, 26 (3). https://www.scielo.br/scielo.php?pid=S010364402015000300216\&script=sci_arttext

Silva, F., Francisco, N. L. S. G., Brum, S. C., Barbosa, C. C. N., \& Soares, L. C. (2016). Antimicrobial activity irrigating solutions to prepare channels biomechanical root face to Enterococcus Faecalis. Brazilian Journal of Surgery and Clinical Research, 15(1), 34-38. https://www.mastereditora.com.br/periodico/20160612_102251.pdf

Silveira, C. M. M., Pimpão, M. V., Fernandes, L. A., Westphalen, V., Cavenago, B.C., \& Carneiro, E. (2019). Influence of Different Irrigation Solutions and Instrumentation Techniques on the Amount of Apically Extruded Debris. European endodontic journal, 4 (3), 122-126. https://www.ncbi.nlm.nih.gov/pmc/articles/PMC7006593/

Siqueira, A. L., Dantas, C. G., Gomes, M. Z., Padilha, F. F., Albuquerque, R. L. C. D., \& Cardoso, J. C. (2014). Study of antibacterial action of hydroalcoholic extract of propolis red on Enterococcus faecalis. Revista de Odontologia da UNESP, 43(6), 359-366. https://www.scielo.br/scielo.php?pid=S1807-25772014000600359\&script=sci_arttext

Souza, F. J. F. (2015). Endodontia passo a passo: Evidências clínicas. In: B. Gomes, P. F. A. Microbiologia Aplicada. Cap. 2, pp. 26-30. Artes Médicas.

Ulusoy, O. I., Savur, I. G., Alaçam, T., \& Çelik, B. (2018). The effectiveness of various irrigation protocols on organic tissue removal from simulated internal resorption defects. International endodontic Journal, 51(1), 1030-1036. https://onlinelibrary.wiley.com/doi/abs/10.1111/iej.12919

Yadav, H. K., Yadav, R. K., Chandra, A., \& Tikku, A. P. (2017). A Scanning Electron Microscopic Evaluation of the Effectiveness of Etidronic Acid, SmearClear and MTAD in Removing the Intracanal Smear Layer. Journal of Dentistry, 18(2), 118-126. https://www.ncbi.nlm.nih.gov/pmc/articles/PMC5457522/

Zandi, H., Rodrigues, R. C. V., Kristoffersen, A. K., Ørstavik, D., Roças, I. N., \& Siqueira, J. F. Jr. (2016). Antibacterial effectiveness of 2 roots canal irrigants in root-filled teeth with infection: a randomized clinical trial. Journal of endodontics, 42(9), 1307-1313. https://www.jendodon.com/article/S00992399(16)30341-7/fulltext 